\title{
On the infrared behaviour of QCD Green functions in the Maximally Abelian gauge
}

\author{
Reinhard Alkofer* \\ Institut für Physik, Karl-Franzens-Universität, Universitätsplatz, 5, A-8010 Graz, Austria \\ E-mail: reinhard.alkofer@uni-graz.at
}

\author{
Markus Q. Huber \\ Institut für Kernphysik, Technische Universität Darmstadt, Schlossgartenstr. 9, 64289 Damstadt, \\ Germany \\ E-mail: markus.huberephysik.tu-darmstadt.de
}

Valentin Mader

Institut für Physik, Karl-Franzens-Universität, Universitätsplatz, 5, A-8010 Graz, Austria

E-mail: valentin.mader@uni-graz.at

\section{Andreas Windisch}

Institut für Physik, Karl-Franzens-Universität, Universitätsplatz 5, A-8010 Graz, Austria

E-mail: andreas.windisch@uni-graz.at

\begin{abstract}
Functional equations like exact renormalisation group and Dyson-Schwinger equations have contributed to a better understanding of non-perturbative phenomena in quantum field theories in terms of the underlying Green functions. In Yang-Mills theory especially the Landau gauge has been used, as it is the most accessible gauge for these methods. In the maximally Abelian gauge first results have been obtained which are very encouraging because Abelian infrared dominance has been found: The Abelian part of the gauge field propagator is enhanced at low momenta and thereby dominates the dynamics in the infrared. Also the ambiguity of two different types of solutions (decoupling and scaling) exists in both gauges. It is demonstrated how the two solutions are related in the maximally Abelian gauge. As in all two-point Dyson-Schwinger equations of the MAG the infrared dominant diagrams are sunset diagrams, in addition, a BPHZ regularisation and renormalisation of a test system with a sunset-like diagram is presented.
\end{abstract}

International Workshop on QCD Green's Functions, Confinement and Phenomenology 5-9 September 2011

Trento, Italy

\footnotetext{
* Speaker.
} 


\section{Motivation: The dual superconductor picture of confinement}

A generally agreed upon explanation of confinement is still lacking. As a matter of fact, the last confinement conference has hosted a panel discussion with the title "What don't we know about confinement?" [1]. Actually it turns out that there are many unanswered questions, and we do not even know if we already know all questions. Within the many suggested different scenarios the dual superconductor picture [2,3] plays a special role. It has been with us for now almost forty years due to its appealing physical nature of an explanation of confinement. Nevertheless, it proved to be very hard to substantiate this scenario.

The dual superconductor picture exploits an analogy to the Meißner-Ochsenfeld effect in typeII superconductors: The vacuum is assumed to contain condensed chromomagnetic monopoles ${ }^{1}$ which squeeze the chromoelectric field lines between two colour charges into a flux tube. These vortex like structures can be identified best after an appropriate choice of gauge which singles out the Cartan subalgebra of the gauge group's Lie algebra. Several such partial gauge fixings have been introduced [6]. The most widely used one is the maximally Abelian gauge (MAG): In this gauge the components of the gauge field outside the Cartan subalgebra are minimized. As the corresponding generators are off-diagonal matrices, the corresponding gluon field components are called off-diagonal gluon fields in contrast to the diagonal ones, which correspond to the gluon field in the Cartan subalgebra.

If the dual superconductor picture is correct the diagonal gluons must dominate the infrared (IR) properties of a Yang-Mills theory in the confinement phase. This follows directly from the fact that also the chromomagnetic monopoles live in the Cartan subalgebra [7]. A detailed understanding of this so-called hypothesis of Abelian IR dominance is missing. In terms of MAG Yang-Mills Green functions one possible realization is an IR finite behaviour for all three propagators of the theory (diagonal and off-diagonal gluons as well as ghosts) such that the largest value is assumed for the diagonal gluon propagator. Attributing to the inverse of the propagator at vanishing momentum a screening mass, $m^{2} \propto D^{-1}(0)$, it is evident that in the deep IR the off-diagonal gluon fields and the ghost decouple from the IR dynamics which is then dominated by the diagonal gluons. Such a behaviour has been found in lattice Monte-Carlo studies (see, e.g., refs. [8, 9]), in the refined Gribov-Zwanziger framework [10, 11], and in a so-called replica model of the MAG [12]. Another possible realization of Abelian IR dominance would be a diverging diagonal gluon propagator. Such a behaviour turns out to be a potential solution of functional equations by employing an IR scaling analysis of the MAG $[13,14]$. This is reminiscent of the coexistence of a scaling solution with an one-parameter family of decoupling solutions for functional equations in other gauges. Such a multitude of solutions is also known from the Coulomb [15, 16] and the Landau gauges $[17,18]$.

To fix the notation we will briefly summarize the concept of the MAG in the following section. We will then discuss the scaling analysis as well as the coexistence of solutions. As these results point towards a dominance of two-loop terms in the propagator Dyson-Schwinger equations, we will report on our efforts to regularize sunset and squint diagrams such that they can be efficaciously included in a self-consistent solution of Dyson-Schwinger equations.

\footnotetext{
${ }^{1}$ Chromomagnetic monopoles are gauge independent objects [4], nevertheless, their detection in lattice configurations in general depends on the choice of gauge [5].
} 


\section{Fundamentals of the maximally Abelian gauge}

To define the MAG of an SU(N) Yang-Mills theory one needs to split the gauge field into diagonal and off-diagonal components:

$$
A_{\mu}=T^{i} A_{\mu}^{i}+T^{a} B_{\mu}^{a},
$$

where the $T^{i}$ are the $N-1$ generators of the Cartan subalgebra of $S U(N)$ with $\left[T^{i}, T^{j}\right]=0$. In the physically interesting case of $S U(3)$ the diagonal generators are $T^{3}$ and $T^{8}$. It is a widely employed notation to use the indices $i, j, \ldots$ for diagonal generators only, and $a, b, \ldots$ for off-diagonal ones. The indices $r, s, \ldots$ are used if both types, diagonal and off-diagonal, generators are present within one relation. Furthermore, we use $A$ for the diagonal and $B$ for the off-diagonal gluon fields.

The herewith introduced splitting of gluon fields has direct consequences for the possible interactions between the now different components of the gluon fields. Starting from the standard commutation relation of the generators,

$$
\left[T^{r}, T^{s}\right]=i f^{r s t} T^{t},
$$

one can directly see that only three off-diagonal or two off-diagonal and one diagonal fields can interact. For the gauge group $S U(2)$ there are more restrictions because there exist only two offdiagonal generators and therefore the first possibility cannot be realized. The number of interaction vertices in $S U(N>2)$ is then larger than in $S U(2)$. In summary the pure Yang-Mills part has the following types of interaction vertices: $A B B, A A B B$ and $B B B B$ for $S U(2)$ and additionally $B B B$ and $A B B B$ for $S U(N>2)$.

The MAG is tuned to minimize the norm of the off-diagonal gluon fields in order to make the effect of the diagonal part most pronounced. The extrema of the functional

$$
\frac{1}{2} \int d x B_{\mu}^{a}(x) B_{\mu}^{a}(x)
$$

are taken if $D_{\mu}^{a b} B_{\mu}^{b}=0$. Hereby the covariant derivative contains only the diagonal gluon field:

$$
D_{\mu}^{a b}:=\delta^{a b} \partial_{\mu}+g f^{a b i} A_{\mu}^{i}
$$

To employ functional methods the remaining $U(1)^{N-1}$ symmetry of the action is conveniently fixed to the Landau gauge, $\partial_{\mu} A_{\mu}^{i}=0$. Since the field $A^{i}$ is Abelian the corresponding Faddeev-Popov ghosts decouple and only the ghosts of the non-diagonal sector need to be taken into account. Hereby, a quartic ghost self-interaction term in the action is required to maintain renormalisability $[19,20]$. The interactions stemming from the gauge fixing and the renormalisability requirement are therefore $A c c, A A c c, B B c c, c c c c, B c c$, and $A B c c$. The two latter interaction vertices vanish for $S U$ (2). This finally leads to the complete action of Yang-Mills theory in MAG:

$$
\begin{aligned}
S_{M A G}=\int & d x\left(\frac{1}{4} F_{\mu \nu}^{i} F_{\mu \nu}^{i}+\frac{1}{4} F_{\mu \nu}^{a} F_{\mu \nu}^{a}+\bar{c}^{a} D_{\mu}^{a b} D_{\mu}^{b c} c^{c}-g f^{b c d} \bar{c}^{a} D_{\mu}^{a b} B_{\mu}^{c} c^{d}-g^{2} \zeta f^{a b i} f^{c d i} B_{\mu}^{b} B_{\mu}^{c} \bar{c}^{a} c^{d}+\right. \\
& +\frac{1}{2 \alpha}\left(D_{\mu}^{a b} B_{\mu}^{b}\right)^{2}+\frac{\alpha}{8} g^{2} f^{a b c} f^{a d e} \bar{c}^{b} c^{c} \bar{c}^{d} c^{e}-\frac{1}{2} g f^{a b c}\left(D_{\mu}^{a d} B_{\mu}^{d}\right) \bar{c}^{b} c^{c}+ \\
& \left.+\frac{1}{4} g^{2} \alpha f^{a b i} f^{c d i} \bar{c}^{a} \bar{c}^{b} c^{c} c^{d}+\alpha \frac{1}{8} g^{2} f^{a b c} f^{a d e} \bar{c}^{b} \bar{c}^{c} c^{d} c^{e}+\frac{1}{2 \xi}\left(\partial_{\mu} A_{\mu}^{i}\right)^{2}\right),
\end{aligned}
$$


where $\alpha$ is the gauge fixing parameter of the off-diagonal and $\xi$ the one of the diagonal sector.

For an action containing three fields with eleven interaction vertices the derivation of its Dyson-Schwinger equations (DSEs) or functional renormalisation group equations (FRGEs) is very lengthy. To this end we employ the computer algebra package DoFun [21], the successor of DoDSE [22] $]^{2}$. Two new features of DoFun as compared to DoDSE are the inclusion of FRGEs and the derivation of the Feynman rules from a given action. In addition, the complete algebraic form of the integrands can now be obtained so that computations directly with Mathematica are possible. This was very helpful for the calculations of the IR leading diagrams of the MAG [14] and the Gribov-Zwanziger action [24]. For the latter the application of DoFun helped to identify a unique solution [25]: Only with the help of a computer algebra system one is able to show that one of the two possible scaling solutions found in [24] does not yield a numerical solution for the infrared exponents and can thus not be realized.

\section{Infrared analysis}

Functional methods provide equations for the fully dressed Green functions. Therefore these equations are applicable to the IR regime of Yang-Mills theories and provide, at least in principle, information about the IR behaviour of the theory [26, 27]. The gauge investigated best is the Landau gauge. Here two types of solutions have emerged from the analysis of functional equations: The scaling solution $[18,24,26,27,28,29,30,31,32,33,34]$ and the one-parameter family of decoupling solutions $[17,18,32,35,36]$. The former is characterized by an IR vanishing gluon propagator, an IR enhanced ghost propagator and IR enhanced three- and four-gluon vertex functions, whereas the latter possesses only IR finite dressing functions except for the gluon dressing [32]. Both solutions are connected by the choice of a renormalisation condition which is a needed input to solve the equations $[17,18]$. The difference only affects the deep IR behaviour of the propagators and vertex functions, while all solutions agree for intermediate momenta well below the scale where perturbation becomes valid ${ }^{3}$. Up to now no calculation exists where these two types of solutions generate a difference for physical quantities: In ref. [37] a confining Polyakov loop potential was derived for both types, the confinement and the chiral transition temperatures were found to coincide for scaling and decoupling [38], and there are hints that even meson masses are independent of this choice [39]. This substantiates the interpretation of this additional type of boundary condition as a non-perturbative gauge fixing parameter as in the Landau- $B$ gauges of ref. [40]. It is worthwhile to mention that this dichotomy also exists in Coulomb gauge $[15,16]$.

One major result to be presented here is that in the MAG one also obtains this two qualitatively different types of solutions, and that there exists a similar connection between them. For the IR analysis we make the ansatz that all dressing functions follow a power law in the deep IR, e.g., for a propagator one uses the ansatz $D(p)=c\left(p^{2}\right) / p^{2}$ and $c^{I R}=d \cdot\left(p^{2}\right)^{\delta}$, where $\delta$ is the related infrared exponent (IRE). As we are in a first step interested in the qualitative behaviour we want to determine the different IREs. Therefore we exploit the very welcome possibility to shift the analysis to the

\footnotetext{
${ }^{2}$ Recently, the program CrasyDSE has become available [23]. It provides a framework for solving DSEs numerically and can directly be combined with DoFun.

${ }^{3}$ As perturbation theory is included in the functional equations all solutions, of course, agree on the multi-GeV scale.
} 
level of the IREs only. This type of IR analysis uses the fact that all integrals are dominated by low momenta if the external momenta are low. Thus, for the purpose of obtaining equations for the IREs one can replace all propagators and vertices by the corresponding IR expressions [34, 41]. Then one counts all exponents of momenta and calculates by this procedure the IRE of any given diagram.

The most elaborate method to derive the scaling relations of the IREs is the combined use of Dyson-Schwinger and Functional Renormalisation Group equations. This was first done for the Landau gauge in Refs. [42, 43]. This method can be generalized rather generically so that one can reduce the infinitely many equations for the IREs to a rather small number of relations between the IREs $[13,14]$.

Without loss of generality, for the MAG at $\xi=0$ the propagators can be parametrized as

$$
\begin{aligned}
D_{A}^{i j}\left(p^{2}\right) & =\delta^{i j} \frac{c_{A}\left(p^{2}\right)}{p^{2}}\left(g_{\mu v}-\frac{p_{\mu} p_{v}}{p^{2}}\right) \\
D_{B}^{a b}\left(p^{2}\right) & =\delta^{a b} \frac{c_{B}\left(p^{2}\right)}{p^{2}}\left(g_{\mu v}-(1-\alpha) \frac{p_{\mu} p_{v}}{p^{2}}\right), \\
D_{c}^{a b}\left(p^{2}\right) & =-\delta^{a b} \frac{c_{c}\left(p^{2}\right)}{p^{2}}
\end{aligned}
$$

The following notation for the power laws of the dressing functions in the IR is used:

$$
c_{A}\left(p^{2}\right) \stackrel{p^{2} \rightarrow 0}{=} d_{A} \cdot\left(p^{2}\right)^{\delta_{A}}, \quad c_{B}\left(p^{2}\right) \stackrel{p^{2} \rightarrow 0}{=} d_{B} \cdot\left(p^{2}\right)^{\delta_{B}}, \quad c_{c}\left(p^{2}\right) \stackrel{p^{2} \rightarrow 0}{=} d_{c} \cdot\left(p^{2}\right)^{\delta_{c}} .
$$

As it is not possible to set the gauge fixing parameter of the off-diagonal part directly to zero the longitudinal part of the off-diagonal propagator could in principle acquire an own dressing function. However, a careful analysis reveals that no new IRE is generated. Based on this we assume in the following infrared analysis only one common dressing function for both tensors.

The main result is that there exists only one consistent scaling relation. It reads [13]:

$$
\kappa_{M A G}:=-\delta_{A}=\delta_{B}=\delta_{c} \geq 0 .
$$

This result for the IREs especially implies that the diagonal gluons are IR enhanced and the off-diagonal ones are IR suppressed. An upper bound for the parameter $\kappa_{M A G}$ can be obtained by demanding well-defined Fourier transformations of the propagators [31]: $\kappa_{M A G}<1$. The IREs for the vertex functions are such that these functions become more IR divergent when the number of off-diagonal legs increases [13]. This applies to the MAG in $S U(2)$ and $S U(N>2)$. It is an interesting result in itself that no differences for the IREs have been found. The additional interactions for larger gauge groups do neither spoil the $S U(2)$ relations nor do they allow an additional solution.

As understood by now the treatment of the bare two-point functions play a decisive role in the respective Dyson-Schwinger equations. To allow for a scaling solution at least one of the zero-momentum values of these Born terms has to be canceled by quantum effects. This is well known for the scaling solution in Landau gauge: The bare ghost two-point function vanishes due to the choice of a corresponding renormalisation condition [28, 18, 44]. To put no prejudice on 
the system we allow all Green functions to become IR divergent. However, it turns out that the only consistent solution is the one with an IR enhanced diagonal gluon propagator. As a first remark, we want to emphasize that we count this as strong evidence that also in the decoupling solution the diagonal gluon propagator is the IR dominant quantity. Second, we point out that the value of the diagonal two-point function at zero momentum can serve as an additional gauge fixing parameter as the ghost two-point function does in the Landau- $B$ gauges of Ref. [40]. To be precise, an IR finite diagonal gluon propagator implies IR finite off-diagonal propagators. The smaller the value in the renormalisation condition becomes the closer one is to the scaling solution.

In the MAG, the reason for this entanglement is the quartic interactions between diagonal and off-diagonal fields. If the diagonal gluon propagator is IR finite, the corresponding tadpole diagrams are proportional to the inverse of the IR value of the diagonal gluon propagator. Via this mechanism IR finiteness of all two-point dressing functions is implied. Tuning the zero momentum value of the diagonal gluon propagator to zero by an appropriate renormalisation condition switches then to the scaling solution. Note that this is a different situation than in Landau (or Coulomb) gauge: There is no quartic interaction between the ghost and the gluon in the Landau gauge, and consequently an IR finite gluon propagator does not enforce an IR finite ghost propagator.

This IR analysis is, of course, not compelling evidence that the scaling solution exists (at least, in the mathematical sense). A numerical calculation is, however, enormously more complicated than in Landau gauge. This is implied by the nature of the MAG scaling solution: Two-loop diagrams are the IR leading ones. Therefore, for a consistent numerical treatment a more elaborated truncation scheme has to be developed. Continuity arguments then imply that even for the MAG decoupling solution a straightforward one-loop truncation likely will miss the essential terms.

\section{Determination of infrared exponents}

Before a numerical solution of the MAG functional equations is attempted it is instructive to calculate the parameter $\kappa_{M A G}$ and therewith the IREs ${ }^{4}$. To do this one can restrict oneself to the IR leading diagrams. Again an ambiguity is found because the role of the squint diagrams cannot be determined by the IR analysis alone. In the first step we restricted ourselves to the sunset diagrams, and the accordingly projected equations reduce in the IR to

$$
\begin{aligned}
& d_{A}^{-1}=-X_{A A B B}^{A}\left(p^{2}, \kappa_{M A G}\right) d_{A} d_{B}^{2}-X_{A A c c}^{A}\left(p^{2}, \kappa_{M A G}\right) d_{A} d_{c}^{2} \\
& d_{B}^{-1}=-X_{A A B B}^{B}\left(p^{2}, \kappa_{M A G}\right) d_{A}^{2} d_{B} \\
& d_{c}^{-1}=-X_{A A c c}^{c}\left(p^{2}, \kappa_{M A G}\right) d_{A}^{2} d_{c} .
\end{aligned}
$$

The quantities $X\left(p^{2}, \kappa_{M A G}\right)$ denote the sunset integrals without the coefficients from the propagator power laws. The superscript gives the corresponding Dyson-Schwinger equation, and the subscript the bare vertex contained in the diagram. Using the invariant combinations $I_{1}:=d_{A}^{2} d_{B}^{2}$ and $I_{2}:=$ $d_{A}^{2} d_{c}^{2}$ the three equations can be combined:

$$
1=\frac{X_{A A B B}^{A}\left(p^{2}, \kappa_{M A G}\right)}{X_{A A B B}^{B}\left(p^{2}, \kappa_{M A G}\right)}+\frac{X_{A A c c}^{A}\left(p^{2}, \kappa_{M A G}\right)}{X_{A A c c}^{c}\left(p^{2}, \kappa_{M A G}\right)} .
$$

\footnotetext{
${ }^{4}$ In this calculation the use of the program DoFun $[22,21]$ was vital since the intermediate expressions appearing there are many pages long.
} 

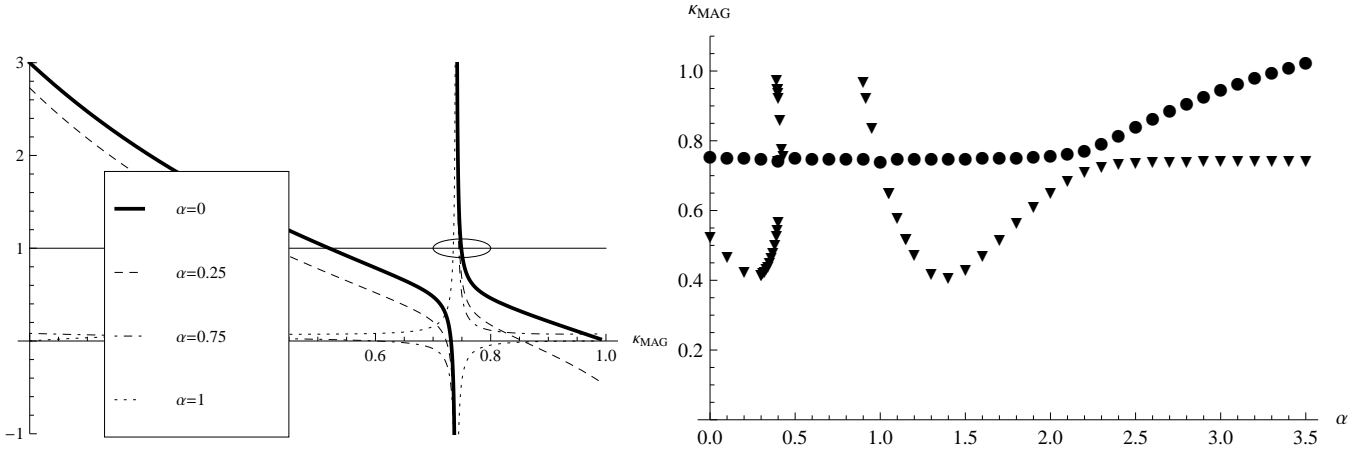

Figure 1: In the left panel the r.h.s. of eq. (4.4) is shown for several values of the gauge fixing parameter $\alpha$. Crossings with the horizontal line represent solutions for $\kappa_{M A G}$. In the right panel the two solution branches are displayed as function of $\alpha$.

With $X\left(p^{2}, \kappa_{M A G}\right)$ being known this equation yields the solution(s) for $\kappa_{M A G}$. For their computation the dressed four-point functions are needed as input. Their power law behaviour is known to be IR constant, but the corresponding tensor structures are not available. We employ the reasonable assumption that the tree-level structures reflect the general properties. (For these tree-level expressions see, e.g., [14].) The last unspecified quantity is the gauge fixing parameter $\alpha$ of the off-diagonal part . In the left panel of Fig. 1 the r.h.s. of eq. (4.4) is displayed for several values of $\alpha$. It turns out that there are solutions with $0.7<\kappa_{M A G}<0.8$ for all reasonable values of $\alpha$. The right panel displays this directly: As a function of $\alpha$ there are two solution branches, with one solution always close to $\kappa_{M A G} \approx 0.74$. The independence of $\alpha$ is a surprisingly positive outcome, and it even persists for rather high $\alpha$.

We want to emphasize that obtaining a solution for $\kappa_{M A G}$ is a highly non-trivial result: The IR analysis depends only on the combinatorics of Feynman diagrams whereas the computation of $\kappa_{M A G}$ relies on all the little details such as Lorentz and colour structure.

\section{Renormalisation of two-loop terms in Dyson-Schwinger equations}

As described above the challenging task for a consistent truncation of the Dyson-Schwinger equations of MAG Yang-Mills theory rests on the inclusion of two-loop terms. In this respect it turns out that the renormalisation of the latter (which is already a considerable task in perturbation theory) provides the first serious obstacle for a self-consistent solution. As it is well-known truncations do interfere with renormalisation. This leads to so-called "spurious divergences" as truncation artefacts. These spurious UV divergences have been observed in many practical calculations, and different techniques to identify or even avoid them have been proposed. For example, for the ghostgluon system in Landau gauge the Brown-Pennington-projector [47] can be applied to keep only the renormalisable terms.

In self-consistent calculations on the one-loop level it has turned out that a quite successful technique consists in applying a momentum subtraction scheme including only the finite and logarithmically divergent self-energies. The renormalisation conditions are imposed onto the dressing functions at the subtraction scale $[48,49]$. Multiplicative renormalisability is then also one of the 
guiding principle for the construction of ansätze for the truncated Green functions [18]. These techniques, however, have only been applied to one-loop truncations ${ }^{5}$.

From perturbation theory we know that the structure of divergences in two-loop terms can be much more complicated then in the one-loop case. UV divergences can now be overlapping and/or nested. In the past many techniques have been developed to remove such UV divergences. Among them are dimensional regularisation and the BPHZ-procedure using Taylor-subtraction [51, $52,53,54]$. Dimensional regularisation belongs to the most spread regularisation procedures in perturbative calculations since it preserves gauge invariance intrinsically. Unfortunately, in selfconsistent calculations this approach is numerically very demanding [55].

The strength of the BPHZ procedure is that it is a clear, well-known and tested method in perturbation theory. It handles overlapping and nested divergences and can render any Feynman diagram finite. In addition, it is free of analytical functions which are numerically expensive to calculate. The general idea to include the BPHZ method into DSE calculations is as follows: Every specific diagram appearing in a (truncated) DSE can be made finite using Zimmermann's forest formula, i.e., all UV divergences are removed, and the integral is then cut-off independent. The renormalisation is then again, as in one-loop calculations, performed in a MOM-scheme.

To exemplify the method we consider a generic propagator DSE. All terms depend on the external momentum $p$, on a renormalisation scale $\mu$ and a cut-off scale $\Lambda$. Given some renormalisation constants $Z_{i}$ one has:

$$
D^{-1}(p ; \mu) p^{2}=Z_{3}(\mu, \Lambda) p^{2}+Z_{1}(\mu, \Lambda) \Pi(p ; \Lambda),
$$

where $D(p ; \mu)$ denotes the propagator dressing function and $\Pi(p ; \Lambda)$ the self-energy term.

Eq. (5.1) is finite by definition. The two factors $Z_{3}$ and $Z_{1}$, however, are totally unknown. They have to be determined in the renormalisation process. The dependence of the self-energy terms on the cut-off is removed by the forest formula which introduces a new scale into the calculation, the subtraction point $s: Z_{1}(\mu, \Lambda) \Pi(p ; \Lambda) \rightarrow Z_{1}(\mu, s) \tilde{\Pi}(p ; s)$.

Imposing multiplicative renormalisability one obtains conditions for the dressing functions of the truncated Green functions. One can then absorb the renormalisation constant $Z_{1}$ in the ansatz for the truncated Green function $[48,49,18]$ and write $Z_{1}(\mu, s) \tilde{\Pi}(p ; s) \rightarrow \tilde{\Pi}(p ; \mu)$.

Having obtained the properly BPHZ regularized DSE one can perform the renormalisation procedure in a MOM scheme. In the last equation the renormalisation constant $Z_{3}$ is the only unknown quantity left. To remove it we subtract at a renormalisation scale $p^{2}=\mu^{2}$ and get

$$
D^{-1}(p ; \mu)=D^{-1}(\mu ; \mu)+\tilde{\Pi}(p ; \mu)-\tilde{\Pi}(\mu ; \mu) .
$$

The self-energy term can now be calculated and gives a definite cut-off independent result. The term $D^{-1}(\mu ; \mu)$ is defined by the renormalisation condition.

To test the procedure we will apply it to the simple DSE-like integral equation with scalar propagators in four dimensions depicted in Fig.2. Its divergences are treated by applying a BPHZ subtraction to its integrand. The regularized integrand is obtained by applying the forest formula to the unregularised integrand: $\tilde{I}_{\Pi}=\sum_{\mathscr{U} \in \mathscr{F}_{\Pi}} \prod_{\xi \in \mathscr{U}}\left(-t^{d(\xi)}\right) I_{\Pi}$. The sum in this equation runs over

\footnotetext{
${ }^{5}$ Two-loop terms have been addressed in ref. [50], but in this study the two-loop terms have been approximated by two one-loop integrals.
} 


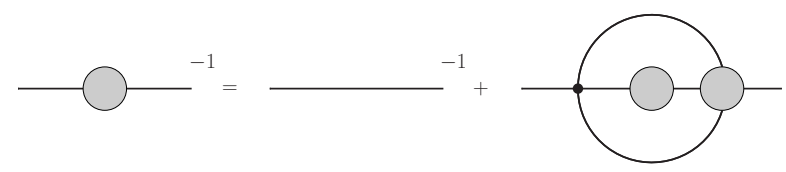

Figure 2: A simple self-consistent equation to test the algorithm described in the text.

all elements of the family of forests $\mathscr{F}_{\Pi}$ of the self-energy diagram $\Pi$, while $\xi$ addresses the subdiagrams appearing in the subsets $\mathscr{U}$. The operator $t^{d(\xi)}$ is the Taylor subtraction operator up to the order of the superficial degree of divergence of the subelement $\xi$, and is defined by $t^{n}=\sum_{i=0}^{n} \frac{p^{n}}{n !}\left[\frac{\partial^{n}}{\partial p^{n}}\right]_{p^{2}=0}$. For the sunset-diagram we then obtain a finite and cut-off independent integrand given by $\tilde{I}_{\Pi}=\left(1-t^{d(\Pi)}\right) I_{\Pi}$.

Next, we have to determine the superficial degree of divergence which is given by two (quadratically divergent). We thus have to Taylor-subtract up to second order to get the regularized integrand. We choose a particular momentum routing through the three propagators of the diagram from which only the middle one is considered dressed. The upper and the lower propagator carry a momentum of $\frac{1}{2} p+q$ and $\frac{1}{2} p+k$ respectively, where all momenta flow from the left to the right and where $p$ is the external momentum. The propagator in the middle carries thus a momentum of $-k-q$. For the 4-scalar vertex we use an ansatz leading then to the unregularised integral

$$
\begin{aligned}
\Pi(p, \Lambda)=\frac{1}{3 !} \int_{\Lambda} \frac{d^{4} k}{(2 \pi)^{4}} \int_{\Lambda} \frac{d^{4} q}{(2 \pi)^{4}} & {\left[\frac{1}{\left[\left(\frac{1}{2} p+k\right)^{2}+\eta^{2}\right]} \cdot \frac{Z(-k-q)}{\left[(k+q)^{2}+\eta^{2}\right]} \cdot \frac{1}{\left[\left(\frac{1}{2} p+q\right)^{2}+\eta^{2}\right]}\right.} \\
\times & \underbrace{\left.g^{2} \frac{1}{Z_{1}} \cdot \frac{1}{Z\left(\frac{1}{2} p+k\right)} \cdot \frac{1}{Z\left(\frac{1}{2} p+q\right)}\right] .}_{\Gamma_{4 s}}
\end{aligned}
$$

Next, we have to Taylor-subtract twice on the integrand of this equation. Hereby we only have to take the $p$ dependent part into account. Note that the contribution of the vertex, $\Gamma_{4 s}$, is relevant and important for multiplicative renormalisability. For the numerical treatment we switch to hyperspherical coordinates and perform three angular integrations trivially. With the resulting quite lengthy expression at hand we solve the equation depicted in Fig. 2 self-consistently. We checked successfully that multiplicative renormalisability is achieved, see Fig. 3 for the comparison of two solutions with different renormalisation scales.

After having successfully developed and tested this technique we employ it right now for the gluon sunset diagram in the gluon propagator DSE in the Landau gauge and to the IR leading sunsets in the MAG.

\section{Summary}

To shed light on the relation between different confinement mechanisms we have studied functional equations in the MAG. Our results provide further evidence for Abelian IR dominance, especially for an IR dominant diagonal gluon propagator and IR suppressed off-diagonal degrees of freedom. We have also found the dichotomy of a scaling versus decoupling solutions known from other gauges, and discussed its origin in the MAG: The two types of solutions are related via the 


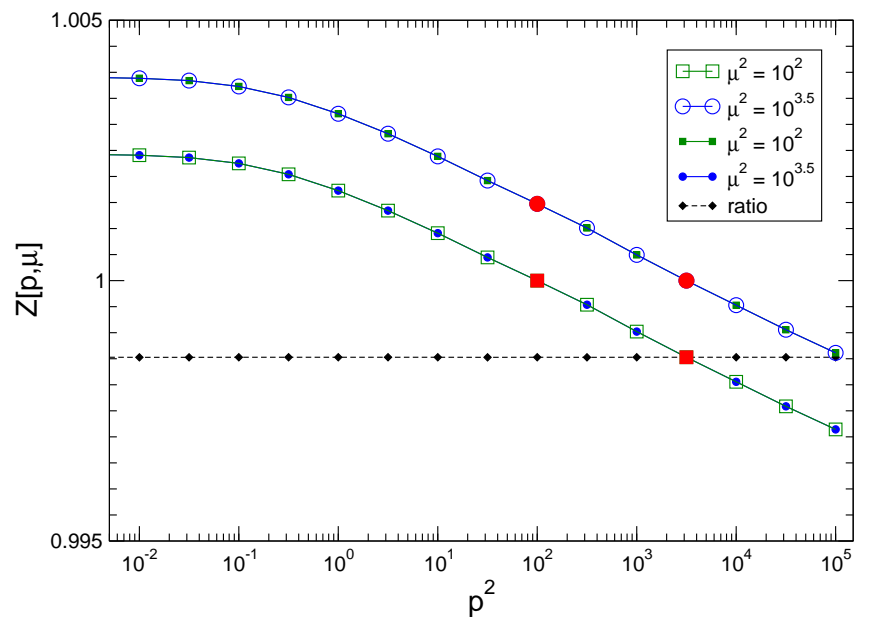

Figure 3: Solution of the equation depicted in Fig. 2. Multiplicative renormalisability is evident as the two obtained solutions differ only by the constant ratio. The renormalisation points are marked in red.

zero momentum value of the diagonal gluon two-point function which is subject to a renormalisation condition.

In order to elucidate this behaviour further we have started the process of numerically solving truncated functional equations keeping the likely IR dominant two-loop terms. In a first step we developed and tested a self-consistent two-loop renormalisation based on BPHZ regularisation. Such a technique is required for solving the DSEs of the MAG which will hopefully shed more light on the confinement mechanism.

\section{Acknowledgments}

We thank the organizers of the workshop for providing such a pleasant and stimulating environment where so many interesting and fruitful discussions were possible. We thank Mario Mitter for helpful discussions, and we are grateful to Natalia Alkofer for a critical reading of the manuscript. Financial support by the FWF project W1203 is gratefully acknowledged. MQH is supported by the Alexander-von-Humboldt foundation.

\section{References}

[1] R. Alkofer, D. Diakonov, J. Pawlowski, H. Reinhardt, V. Zakharov, D. Zwanziger and J. Greensite, AIP Conf. Proc. 1343 (2011) 17 [arXiv:1012.3192 [hep-th].

[2] S. Mandelstam, Phys. Rept. 23 (1976) 245.

[3] G. 't Hooft. Rapporteur's talk given at Int. Conf. on High Energy Physics (Palermo, Italy, Jun 23-28, 1975).

[4] C. Bonati, A. Di Giacomo, L. Lepori and F. Pucci, Phys. Rev. D 81 (2010) 085022 [arXiv:1002.3874 [hep-lat]]. 
[5] C. Bonati, A. Di Giacomo and M. D’Elia, Phys. Rev. D 82 (2010) 094509 [arXiv:1009.2425 [hep-lat]].

[6] G. 't Hooft, Nucl. Phys. B 190 (1981) 455.

[7] Z. F. Ezawa and A. Iwazaki, Phys. Rev. D 25 (1982) 2681.

[8] V. G. Bornyakov, M. N. Chernodub, F. V. Gubarev, S. M. Morozov and M. I. Polikarpov, Phys. Lett. B 559 (2003) 214 [hep-lat/0302002].

[9] T. Mendes, A. Cucchieri, A. Maas and A. Mihara, arXiv:0809.3741 [hep-lat].

[10] M. A. L. Capri, V. E. R. Lemes, R. F. Sobreiro, S. P. Sorella and R. Thibes, Phys. Rev. D 77 (2008) 105023 [arXiv:0801.0566 [hep-th]].

[11] M. A. L. Capri, A. J. Gomez, M. S. Guimaraes, V. E. R. Lemes and S. P. Sorella, J. Phys. A A 43 (2010) 245402 [arXiv:1002.1659 [hep-th]].

[12] M. A. L. Capri, A. J. Gomez, M. S. Guimaraes, V. E. R. Lemes, S. P. Sorella and D. G. Tedesco, arXiv:1110.4395 [hep-th].

[13] M. Q. Huber, K. Schwenzer and R. Alkofer, Eur. Phys. J. C68 (2010) 581 [arXiv:0904.1873 [hep-th]]; PoS FACESQCD (2011) 001 [arXiv:1103.0236 [hep-th]].

[14] M. Q. Huber, PhD thesis [arXiv:1005.1775 [hep-th]].

[15] A. P. Szczepaniak and E. S. Swanson, Phys. Rev. D 65 (2002) 025012 [hep-ph/0107078].

[16] D. Epple, H. Reinhardt, W. Schleifenbaum and A. P. Szczepaniak, Phys. Rev. D 77 (2008) 085007 [arXiv:0712.3694 [hep-th]].

[17] P. Boucaud, J-P. Leroy, A. L. Yaouanc, J. Micheli, O. Pene and J. Rodriguez-Quintero, JHEP 0806 (2008) 012 [arXiv:0801.2721 [hep-ph]].

[18] C. S. Fischer, A. Maas and J. M. Pawlowski, Annals Phys. 324 (2009) 2408 [arXiv:0810.1987 [hep-ph]].

[19] H. Min, T. Lee and P. Y. Pac, Phys. Rev. D 32 (1985) 440.

[20] M. A. L. Capri, R. F. Sobreiro and S. P. Sorella, Phys. Rev. D 73 (2006) 041701 [hep-th/0512096].

[21] M. Q. Huber and J. Braun, arXiv:1102.5307 [hep-th].

[22] R. Alkofer, M. Q. Huber and K. Schwenzer, Comput. Phys. Commun. 180 (2009) 965 [arXiv:0808.2939 [hep-th]].

[23] M. Q. Huber and M. Mitter, arXiv:1112.5622 [hep-th].

[24] M. Q. Huber, R. Alkofer and S. P. Sorella, Phys. Rev. D 81 (2010) 065003 [arXiv:0910.5604 [hep-th]].

[25] M. Q. Huber, R. Alkofer and S. P. Sorella, AIP Conf. Proc. 1343 (2011) 158 [arXiv:1010.4802 [hep-th]].

[26] R. Alkofer and L. von Smekal, Phys. Rept. 353 (2001) 281 [hep-ph/0007355].

[27] C. S. Fischer, J. Phys. G 32 (2006) R253 [hep-ph/0605173].

[28] L. von Smekal, A. Hauck and R. Alkofer, Annals Phys. 267 (1998) 1 [Erratum-ibid. 269 (1998) 182] [hep-ph/9707327].

[29] P. Watson and R. Alkofer, Phys. Rev. Lett. 86 (2001) 5239 [arXiv:hep-ph/0102332]. 
[30] J. M. Pawlowski, D. F. Litim, S. Nedelko and L. von Smekal, Phys. Rev. Lett. 93 (2004) 152002 [hep-th/0312324].

[31] C. Lerche and L. von Smekal, Phys. Rev. D 65 (2002) 125006 [hep-ph/0202194].

[32] R. Alkofer, M. Q. Huber and K. Schwenzer, Phys. Rev. D 81 (2010) 105010 [arXiv:0801.2762 [hep-th]].

[33] N. Alkofer and R. Alkofer, Phys. Lett. B 702 (2011) 158 [arXiv:1102.2753 [hep-th]]; PoS FACESQCD (2011) 043 [arXiv:1102.3119 [hep-th]]; arXiv:1112.4483 [hep-th].

[34] R. Alkofer, C. S. Fischer and F. J. Llanes-Estrada, Phys. Lett. B 611 (2005) 279 [Erratum-ibid. 670 (2009) 460] [hep-th/0412330].

[35] D. Dudal, S. P. Sorella, N. Vandersickel and H. Verschelde, Phys. Rev. D 77 (2008) 071501 [arXiv:0711.4496 [hep-th]].

[36] A. C. Aguilar, D. Binosi and J. Papavassiliou, Phys. Rev. D 78 (2008) 025010 [arXiv:0802.1870 [hep-ph]].

[37] J. Braun, H. Gies and J. M. Pawlowski, Phys. Lett. B 684 (2010) 262 [arXiv:0708.2413 [hep-th]].

[38] C. S. Fischer and J. A. Mueller, Phys. Rev. D 80 (2009) 074029 [arXiv:0908.0007 [hep-ph]].

[39] M. Blank, A. Krassnigg and A. Maas, Phys. Rev. D 83 (2011) 034020 [arXiv:1007.3901 [hep-ph]].

[40] A. Maas, Phys. Lett. B 689 (2010) 107 [arXiv:0907.5185 [hep-lat]].

[41] M. Q. Huber, R. Alkofer, C. S. Fischer and K. Schwenzer, Phys. Lett. B 659 (2008) 434 [arXiv:0705.3809 [hep-ph]].

[42] C. S. Fischer and J. M. Pawlowski, Phys. Rev. D 75 (2007) 025012 [hep-th/0609009].

[43] C. S. Fischer and J. M. Pawlowski, Phys. Rev. D 80 (2009) 025023 [arXiv:0903.2193 [hep-th]].

[44] D. Zwanziger, Phys. Rev. D 65 (2002) 094039 [hep-th/0109224].

[45] A. Cucchieri, A. Maas and T. Mendes, Comput. Phys. Commun. 180 (2009) 215 [arXiv:0806.3124 [hep-lat]].

[46] R. Alkofer, C. S. Fischer, H. Reinhardt and L. von Smekal, Phys. Rev. D 68 (2003) 045003 [hep-th/0304134].

[47] N. Brown and M. R. Pennington, Phys. Rev. D 39 (1989) 2723.

[48] C. S. Fischer and R. Alkofer, Phys. Lett. B 536 (2002) 177 [hep-ph/0202202]; R. Alkofer, C. S. Fischer and L. von Smekal, Acta Phys. Slov. 52 (2002) 191 [arXiv:hep-ph/0205125].

[49] C. S. Fischer, PhD thesis [hep-ph/0304233].

[50] J. C. R. Bloch, Few Body Syst. 33 (2003) 111 [hep-ph/0303125].

[51] N. N. Bogoliubov, O. S. Parasiuk, Acta Math. 97 (1957) 227-266.

[52] N. N. Bogolyubov, D. V. Shirkov, Intersci. Monogr. Phys. Astron. 3 (1959) 1-720.

[53] K. Hepp, Commun. Math. Phys. 2 (1966) 301-326.

[54] W. Zimmermann, Commun. Math. Phys. 15 (1969) 208-234.

[55] A. W. Schreiber, T. Sizer and A. G. Williams, Phys. Rev. D 58 (1998) 125014 [hep-ph/9804385]. 